\title{
PERFIL DERMATOGLÍFICO Y SOMATOTÍPICO DE ATLETAS DE LA SELECCIÓN COLOMBIA DE ATLETISMO (VELOCIDAD) PARTICIPANTE EN LOS JUEGOS PANAMERICANOS DE GUADALAJARA, 2011
}

\section{DERMATOGLYPHIC AND SOMATOTYPIC PROFILE A ATHLETES SELECTION OF ATHLETICS COLOMBIAN'S (SPEED) PARTICIPANT IN THE PANAMERICANS GAMES IN GUADALAJARA, 2011}

\author{
Rafael Ernesto Avella ${ }^{1}$, Juan Pablo Medellín ${ }^{2}$
}

\begin{abstract}
${ }^{1}$ Magister en Entrenamiento Deportivo, Instituto Manuel Fajardo; Especialista en Docencia Universitaria, Universidad Militar Nueva Granada, Docente Investigador de la Universidad de Ciencias Aplicadas y Ambientales U.D.C.A, Director del Grupo de Investigación en Actividad Física y Estilos de Vida Saludable; ravella@udca.edu.co; ${ }^{2}$ Estudiante Investigador de la Facultad de Ciencias del Deporte, Universidad de Ciencias Aplicadas y Ambientales U.D.C.A, Integrante del Grupo de Investigación en Actividad Física y Estilos de Vida Saludable; m_juanpablo9@hotmail.com
\end{abstract}

Rev. U.D.C.A Act. \& Div. Cient. 16(1): 17 - 25, 2013

\section{RESUMEN}

Con el objetivo de generar un perfil preliminar dermatoglífico y somatotípico que sirva para la selección de talentos en el atletismo, en modalidades de velocidad, se evaluaron los atletas de la selección Colombia, participante en los Juegos Panamericanos de Guadalajara, en el 2011. El estudio fue descriptivo de corte transversal y la recolección de datos, se llevó acabo previa firma del consentimiento informado. La metodología utilizada para la colecta de dermatoglifos fue CUMMINS \& MIDLO (1963), la edad de la muestra en damas $(n=5)$ fue de $23 \pm 1,4$ y en varones $(n=3)$ de $22,3 \pm 2,5$; la estatura, respectivamente, estuvo entre $1,72 \pm 0,09$ y 1,76 $\pm 0,05$; la masa corporal en mujeres fue de 63,3 $\pm 3,34$ y en hombres de 74,8 $\pm 7,35$. En cuanto a las variables dermatoglíficas y somatotípicas, los resultados en damas fueron: Arco: 0, Presilla: 7,8 $\pm 2,17$, Verticilo: $2,2 \pm 2,17$, D10: 12,2 $\pm 2,17$, SCTL: $130,4 \pm 36,1$, Ectomorfismo: 3,04 \pm 1,43, Mesomorfismo: 3,1 \pm 1,6 y Endomorfismo: 3,25 \pm 0,59; los varones arrojaron los siguientes datos: Arco: 0,67 \pm 1 , Presilla: $6,7 \pm 1,15$, Verticilo: $3 \pm 1,15$, D10: $12 \pm 2$, SCTL: 123 \pm 19,1, Ectomorfismo: 2,05 \pm 0,25, Mesomorfismo: 4,09 $\pm 1,01$ y Endomorfismo: $2,66 \pm 1,28$. Se aprecia que los valores más destacados en dermatoglífia son la predominancia de dibujos Presilla, ausencia de Arcos y un D10 y SCTL medio; el somatotipo que prevalece en los atletas varones es el mesomorfo y en damas se aprecia una similitud de los tres estadios.

Palabras clave: Huellas, genética, talento, corredores, antropometría.

\section{SUMMARY}

In order to generate a preliminary profile somatotypic and dermatoglyphic to serving for the selection of talent in athletics, speed modes, athletes were evaluated Colombia participant selection in the Pan American Games in Guadalajara in 2011, the study was descriptive cross-sectional data collection took place after signing informed consent. The methodology used for the collection of dermatoglyphics was, the age of the sample in women $(n=5)$ was $23 \pm 1,4$ and in males ( $n$ $=3$ ) of 22,3 $\pm 2,5$, respectively height was at 1,72 $\pm 0,09$ and 1,76 $\pm 0,05$, body mass in women was $63,3 \pm 3,34$ and 74,8 $\pm 7,35$ men, in terms of dermatoglyphic variables and outcomes somatotypics ladies were: Arco: 0, Loop: 7,8 $\pm 2,17$, whorl: $2,2 \pm 2,17$, D10: $12,2 \pm 2,17$, SCTL: 130,4 $\pm 36,1$, ectomorphism: $3,04 \pm 1,43$, mesomorphism: 3,1 $\pm 1,6$ and endomorphism: $3,25 \pm 0,59$; males showed the following data: Arco: 0,67 \pm 1 , Clip: 6,7 $\pm 1,15$, whorl: $3 \pm$ 1,15, D10: $12 \pm 2$, SCTL: $123 \pm 19,1$, ectomorphism: 2,05 \pm 0,25, mesomorphism: 4,09 $\pm 1,01$ and endomorphism: 
$2,66 \pm 1,28$. It is seen that the values are more prominent in the predominance of dermatoglyphics Presilla drawings, no arches, and a D10 and SCTL medium; somatotype prevalent in male athletes is the mesomorph, and ladies can see a similarity of the three stages.

Key words: Footprints, genetics, talent, sprinters, antropometry.

\section{INTRODUCCIÓN}

En las últimas décadas se ha prestado mucha atención al tema de selección y de orientación de talentos. Diferentes países han realizado estudios e inversiones para el desarrollo de estos procesos, buscando niños superdotados y talentosos que, por sus habilidades extraordinarias, sean capaces de altas realizaciones, teniendo un potencial de habilidad en alguna área (Marland, 1972).

El talento deportivo está definido como aquel que posee un individuo en determinado momento de la evolución, capaz de combinar las capacidades motoras y psicológicas y que, además, posee unas estructuras anatómicas y fisiológicas que, en conjunto, le dan la posibilidad y el potencial para el logro de altos resultados deportivos, en una práctica concreta (Zatsiorski, 1989; Hahn, 1988; García Manso, 2003).

En el deporte existen variables a tener en cuenta a la hora de seleccionar un posible talento; la herencia cumple un papel vital en el desarrollo sicomotriz y la condición física (Ruiz \& Sánchez, 1997); a su vez, el medio ambiente y la estimulación influyen sobre el desarrollo de las personas.

En el proceso de la detección de talentos es necesario la intervención de un grupo multidisciplinario, que se encargue de una valoración lo más completa posible; estos serán encargados de la aplicación de pruebas, las cuales, determinaran el potencial del individuo para la práctica deportiva (Martínez et al. 2012). Estas metodologías y áreas se pueden resumir en lo que se ha denominado Caracterización (Grosser et al. 1990; Agudelo, 2012; Dantas, 2012) y que contiene evaluaciones en las diferentes ciencias aplicadas al deporte.

En los últimos tiempos, se han generado alternativas para la selección de talentos en deporte, una de estas tiene que ver con la parte genética, siendo de vital importancia, dado que ofrece información sobre las posibilidades funcionales del sujeto (Rover \& Nodari Junior, 2012), con el estudio detallado del fenotipo (conjunto de caracteres), genotipo (constitución genética) y la relación que estos establecen con el medio (Martínez et al. 2012).

La función principal de los genes es proporcionar la infor- mación codificada para la síntesis de las clases de proteínas (contráctiles, de transporte, enzimáticas, inmunes, hormonales, citoesqueléticas, entre otras), base vital en el proceso adaptativo (Bouchard, 2007).

Componentes como la talla y el peso pueden ser pronosticados, a través de las mediciones de los padres, donde existen altas correlaciones estadísticas para dichos pronósticos; factores como la estatura y la resistencia son aportados en mayor porcentaje por la madre y la fuerza y velocidad por el padre, permitiendo, de alguna manera, establecer cierta predisposición para la práctica deportiva (Leiva, 2010).

Los parámetros investigados permiten tener una base científica para la selección de los posibles talentos que puedan realizar trabajos y entrenamientos muy específicos en el campo del deporte y, de esta manera, identificar poblaciones y sectores que tienen más capacidades frente a la realización de ciertas actividades, estableciendo sus aspectos débiles y fuertes, en función del componente físico, lo cual, ayudará en el proceso de planificación del entrenamiento deportivo, potencializando los aspectos genéticamente predispuestos y desarrollando los limitados por los genes (Martínez et al. 2012).

Además de las pruebas y los estudios realizados, se está desarrollando una nueva herramienta para la detección de talentos en el deporte. Se trata de la dermatoglífia, el estudio de las impresiones o reproducciones de los dibujos formados por las crestas en los pulpejos dactilares de las manos (complejo palmar), dedos (tercera falange) y plantas de los pies (Nishioka et al. 2007), comprendiéndose como un marcador genético, por su asociación con las cualidades físicas básicas y tipología de fibras musculares (Ferrão et al. 2004). La identificación papilar, se basa en que los dibujos formados por las crestas digitales, palmares y plantares, formadas entre el tercer (3) al sexto (6) mes de vida intrauterina (Chakraborty, 1991) son perennes, inmutables e infinitamente diversas constituyéndose en un marcador genético (Anton $\&$ de Luis, 2004; Dantas, 2012).

Las impresiones dactilares, se dividen según su dibujo en tres grandes grupos arcos, presillas y verticilos (Figura 1). Los dibujos dactilares pueden ser divididos en subgrupos, con base a diferencias menores que existen entre ellos.

Estudios adelantados por Abramova et al. (2003) permiten establecer que en deportes cíclicos de velocidad a la fuerza, se asocia con los dibujos dactilares arcos y presilla y menor número de crestas. De otro lado, los dibujos dactilares más complejos y con el mayor número de crestas son propios de aquellos deportes con altas exigencias coordinativas. Una posición intermedia en relación con la complejidad de los dibujos dactilares y el número de crestas es propia de los deportes orientados a la resistencia. 


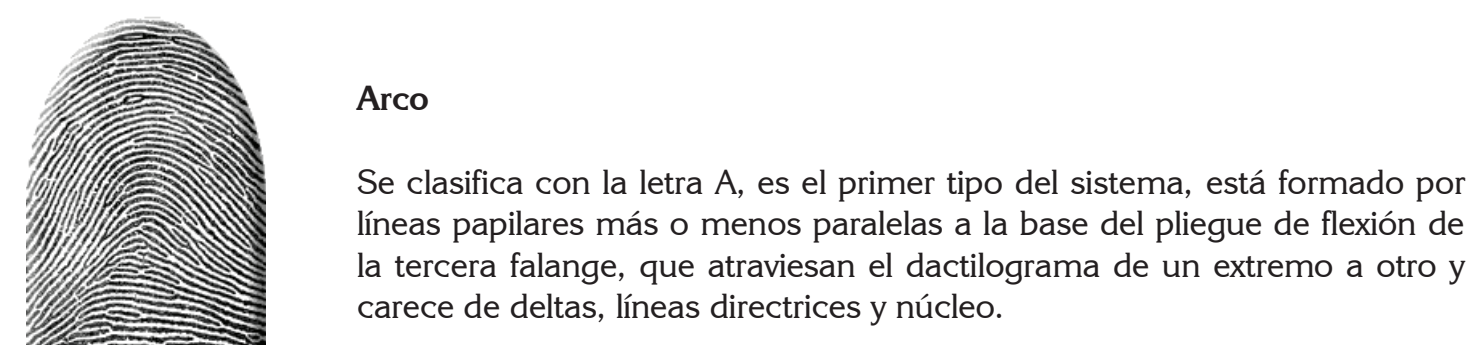

Presilla

Se clasifican con la letra L, está formada por un delta que se encuentra ubicado a la derecha del observador y sus líneas directrices que envuelven a las nucleares se dirigen hacia la izquierda.

\section{Verticilo}

Se clasifica con la letra $\mathrm{V}$, es el último tipo del sistema, esta formado por dos deltas, uno a la derecha y el otro a la izquierda y sus líneas directrices circunscriben al núcleo en diferentes formas.

Figura 1. Impresiones dactilares, según su dibujo arcos, presillas y verticilos (Tomado de Guzmán, 2003; Fonseca et al. 2008; Martínez et al. 2012; Abramova et al. 1996; Nishioka et al. 2007; João \& Fernandes Filho, 2002).

El pronóstico del fenotipo, con base en las manifestaciones del genotipo, conforma la primera posibilidad de modificar la vida de las personas. Cuando se inicia el proceso de selección y de orientación a tempranas edades, la genética ofrece información valedera, acertada y confiable, que permite a los individuos disponer adecuadamente las habilidades, las capacidades y la predisposición que tiene para la realización de una actividad determinada.

Actualmente, la selección deportiva se basa en parámetros morfofuncionales, volitivos y técnicos propios de la ontogénesis: complexión corporal, estado psicológico, cualidades físicas, cambios durante los procesos de crecimiento y maduración biológica (Leiva, 2010). Las investigaciones sobre los criterios genéticos relacionados con las cualidades físicas, generalmente, son de poca aplicación práctica, dado que son en su mayoría invasivos y de difícil consecución.

El somatotipo es una descripción semi - cuantitativa de la forma relativa actual de la composición corporal, la cual, se expresa en tres (3) valores numéricos, representando el componente físico: Endomorfia, Mesomorfia y Ectomorfia (Carter \& Heath, 1990), siendo este utilizado, entre otras, para describir y comparar los atletas en los diferentes niveles de preparación, puntualizar los cambios físicos durante el crecimiento y comparar la forma relativa de hombres y de mujeres (Carvalho et al. 2005), por ende, es uno de los métodos de caracterización deportiva.

Las condiciones físicas reflejan las posibilidades motoras del individuo, determinadas genéticamente y por la oscilación de las diferencias ontogénicas, las cuales, se manifiestan claramente en el entrenamiento de alto nivel (Leiva, 2010).

El atletismo es uno de los deportes en los que la predisposición genética cumple un papel fundamental a la hora de seleccionar los talentos y el entrenamiento cada vez es más exigente y las marcas más difíciles de superar; sin embargo, continua en ascenso y sigue siendo uno de los deportes que más medallas y espectáculo brinda al público. Se divide en 
pruebas de pista y de campo (Silva, 2002); está clasificado dentro de los deportes de tiempo y marca; en la pista están contempladas las pruebas de velocidad o sprints, donde se recorren distancias entre los 100 y los 200 m, en masculino y femenino; en estas la salida es baja, con el apoyo de unos tacos, que rompen la inercia y permiten que el atleta tenga un inicio potente (Casanovas, 2000).

En los últimos años, Colombia ha tenido un gran avance en las pruebas de velocidad, logrando figuraciones en los diferentes eventos internacionales. Dado este progreso de los atletas colombianos, se hace necesario identificar las características dermatoglíficas y somatotípicas, que permitan definir características predominantes en la velocidad en ambos géneros, con el fin de detectar talentos para dicha modalidad.

\section{MATERIALES Y MÉTODOS}

El estudio, se diseñó teniendo en cuenta las reglas de investigación en seres humanos o con muestras de origen humano (Resolución № 008430 de 1993, Resolución No. 01 de 2008. Ministerio de Salud).

Muestra: La muestra estuvo constituida por los participantes en los Juegos Panamericanos Guadalajara 2011 en atletismo, tanto en la rama masculina $(n=3)$ como femenina ( $n$ $=5)$, en las pruebas de velocidad.

Protocolos: Para verificar las características dermatoglíficas fue utilizado el método de Cummins \& Midlo (1963); la recolección de impresiones digitales, se realizó cubriendo de tinta las falanges distales, haciendo una rodada uniforme en las planillas diseñadas para este proceso.

Una vez realizado el dibujo dactilar, ser clasificado y haber realizado el conteo de crestas, se logró determinar la predisposición del sujeto para la realización de un deporte, en especial, aplicando la siguiente fórmula, establecida por Cummins \& Midlo (1963), analizando variables cualitativas (diseños) y cuantitativas (SQTL, D10):

a) Los diseños de las falanges dístales de las manos (Arcos, Presillas y Verticilos). Cantidad de diseños en los dedos de las manos derecha e izquierda Complejidad en los diseños de los diez dedos de las manos (D10), calculada por la ecuación:

$$
\mathrm{D} 10=\Sigma \mathrm{L}+2 \sum \mathrm{W}
$$

Dónde: Arcos (A) 0 puntos, por ello no aparecen en la ecuación; Presillas (L) 1 punto; Verticilos (W) 2 puntos

b) Cantidad de líneas. Se cuenta cada cresta que cruza o toca la línea imaginaria (Línea de Galton), trazada desde el delta hasta el núcleo, sin incluir la cuenta del delta o del núcleo; con base a la cantidad de líneas de todos los dedos de las manos, se calcula SCTL, que es la sumatoria de la cantidad de líneas (Dantas, 2012; Castanhede et al. 2003).

La valoración del somatotipo fue obtenida, a través de la técnica utilizada para medida de los pliegues cutáneos, de las circunferencias y de los diámetros óseos, según las recomendaciones del método de Carter \& Heath (1990).

Para la determinación de las medidas que componen el somatotipo fueron tenidos en cuenta los pliegues cutáneos: subescapular, tricipital, suprailiaco, abdominal, muslo y pierna; utilizando el calibrador de pliegues Lange, calibrado a una presión de $0,2 \mathrm{~mm}$, un rango de $90 \mathrm{~mm}$ y una graduación de 0,1. La medición de diámetros óseos: biestiloideo, biepicondilo humeral (codo) y biepicondilo femoral (rodilla), utilizando un antropómetro, con una longitud de escala de 0-2100 mm, para los perímetros del brazo y para la pantorrilla, una cinta métrica genérica. El peso corporal fue por medio de una báscula digital, marca TANITA Ironman Modelo: BC - 558 y la talla, con un estadiómetro de pared marca.

Para las mediciones de adipometría, se usó el protocolo de Carter (2002), siguiendo las indicaciones, identificando y evaluando los pliegues tricipital, subescapular, suprailíaco, abdominal, muslo, pierna. Todas las mediciones se tomaron en el lado derecho y se esperaron dos segundos para tomar el registro de la medición.

Se determinó el \% de grasa corporal con las siguientes ecuaciones.

Hombres \% de Grasa $=6$ PC x 1,051 + 2,59

Mujeres \% de Grasa $=6$ PC x 0,1548+3,58

En donde los perímetros se tratan de medidas lineales realizadas circunferencialmente a distintos segmentos corporales, como Muslo, Pantorrilla, Antebrazo, Brazo, Cintura, Cadera y Abdomen y los diámetros son las medidas corporales tomadas en sentido horizontal, para las cuales, se utilizó un antropómetro Swiss Made, con una longitud de escala de 0-2100 mm. Los diámetros antropométricos fueron biestiloideo, biepicondílar, del humero y biepicondílar, del fémur.

\section{RESULTADOS Y DISCUSIÓN}

Los datos recogidos durante el presente estudio, se muestran en la tabla 1:

Al comparar los resultados entre el estudio realizado por Dos Santos et al. (2007) con atletas de velocidad de Brasil y los deportistas del presente estudio (Tabla 2), existen gran 
Tabla 1. Resultados estadísticos de las variables analizadas.

\begin{tabular}{|l|c|c|c|c|}
\hline \multirow{2}{*}{} & \multicolumn{2}{|c|}{ Mujeres $(\mathbf{n}=\mathbf{5})$} & \multicolumn{2}{c|}{ Hombres $(\mathbf{n}=\mathbf{3})$} \\
\cline { 2 - 5 } & Media & Desviación & Media & Desviación \\
\hline Edad & 23 & 1,41 & 22,33 & 2,52 \\
\hline Estatura & 1,72 & 0,09 & 1,76 & 0,05 \\
\hline Peso & 63,36 & 3,34 & 74,8 & 7,35 \\
\hline \% Grasa & 13,4 & 0,89 & 8 & 2 \\
\hline Ectomorfia & 3,04 & 1,43 & 2,053 & 0,25 \\
\hline Mesomorfia & 3,1 & 1,60 & 4,09 & 1,01 \\
\hline Endomorfia & 3,25 & 0,59 & 2,66 & 1,28 \\
\hline Arco & 0 & 0,00 & 0,67 & 1 \\
\hline Presilha & 7,8 & 2,17 & 6,7 & 1,15 \\
\hline Verticilo & 2,2 & 2,17 & 3 & 1,15 \\
\hline D10 & 12,2 & 2,17 & 12 & 2 \\
\hline SQTL & 130,4 & 36,15 & 123 & 19,16 \\
\hline
\end{tabular}

similitud en los resultados obtenidos, en el proceso de evaluación de la dermatoglífia y del somatotipo, en cuanto a los varones. Los dibujos dactilares Arco presentan una marcada ausencia en la generalidad de deportistas; los dibujos Presila son un 3\% superiores en atletas colombianos; los Verticilo son iguales en ambas muestras; en el D10, los brasileros dominan un 7,7\%; en el SQTL, se aprecia una diferencia de $5,4 \%$ a favor de los atletas brasileros (menos líneas), donde corroborado con la literatura (Abramova et al. 1990), los predispone más a esfuerzos explosivos e intensos. En las variables de somatotipo, la diferencia más marcada se encuentra en el ectomorfismo, siendo de $25,5 \%$, en la cual, los atletas brasileros son más longilineos, en cuanto al endomorfismo; la diferencia es de $12,1 \%$ y en el mesomorfismo de $0,2 \%$, siendo estas dos últimas poco significativas, pero sin dejarlas de lado, dado que pueden ser determinantes en el deporte de altos logros.

En las damas, los Arcos no presentan ninguna diferencia (ausencia total), mientras en las Presilas, se aprecia una diferencia de $2,5 \%$, siendo las seleccionadas brasileras favorecidas

Tabla 2. Comparación de las variables dermatoglifícas y somatotípicas.

\begin{tabular}{|c|c|c|c|c|}
\hline & Hombres Brasil & Hombres Colombia & Mujeres Brasil & Mujeres Colombia \\
\hline $\mathbf{N}$ & 14 & 3 & 5 & 5 \\
\hline Arco & 0 & 0,67 & 0 & 0 \\
\hline Presila & 6,5 & 6,7 & 8 & 7,8 \\
\hline Verticilo & 3 & 3 & 2 & 2,2 \\
\hline D10 & 13 & 12 & 12 & 12,2 \\
\hline SQTL & 130 & 123 & 105 & 130 \\
\hline Endo & 2,33 & 2,65 & 2,61 & 3,25 \\
\hline Meso & 4,1 & 4,09 & 4,25 & 3,1 \\
\hline Ecto & 2,75 & 2,05 & 4,14 & 3,03 \\
\hline
\end{tabular}

por esta heterogeneidad (mayor dibujos en estas); en los Verticilos, la desigualdad es de $9,1 \%$, teniendo mayor cantidad las colombianas; en el D10 es de 1,6\%, siendo más alto el de las colombianas y el SQTL, es superior 19,2\%, en favor de las brasileras (menos líneas). En cuanto a las variables de la composición corporal, se presentan notables diferencias; en el endomorfismo, la diferencia es de $19,7 \%$, mesomorfismo $27,1 \%$ y en el ectomorfismo de $26,8 \%$, siendo las brasileras más magras, musculosas y más longilíneas.

Los gráficos $1 \mathrm{~A}$ y $\mathrm{B}$ son la normalización de los resultados en ambos géneros, donde los límites se hallaron con las siguientes ecuaciones:

$=($ Dato - Min. $) /($ Max. - Min. $)$
Y, finalmente, se normalizan los límites:

Lim. Inf. $=X-\left((1,96 * S) /\left(\mathrm{n}^{\wedge}(1 / 2)\right)\right.$

Lim. Sup. $=X+\left((1,96 * S) /\left(\mathrm{n}^{\wedge}(1 / 2)\right)\right.$

De la normalización y análisis de los datos por individuo, se genera la gráfica 1A: Mujeres y gráfica 1B: Hombres, que permiten tener un panorama grupal sobre las condiciones de somatotipo y dermatoglifia y establecer un modelo grafico preliminar, para orientar el proceso en la selección de talentos, para pruebas de velocidad.

Al comparar las gráficas obtenidas en el presente estudio con los realizados por Castanhede et al. (2003) (Gráfica 2) en fútbol, Fonseca et al. (2008), en voleibol y Carvalho et al. (2005), en atletismo de resistencia, se aprecia que en to- 


\section{VALORES NORMALIZADOS (Mujeres)}

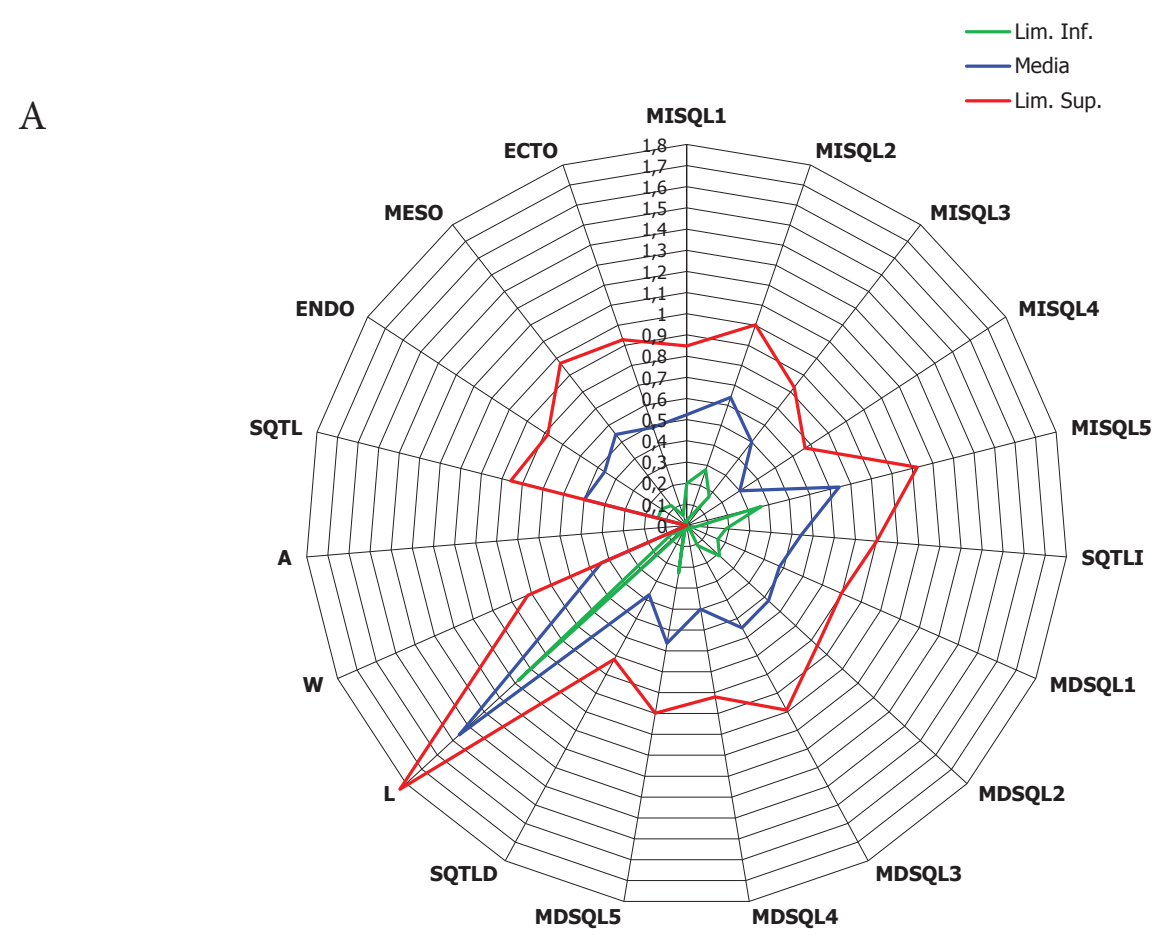

VALORES NORMALIZADOS (Hombres)

$\mathrm{B}$

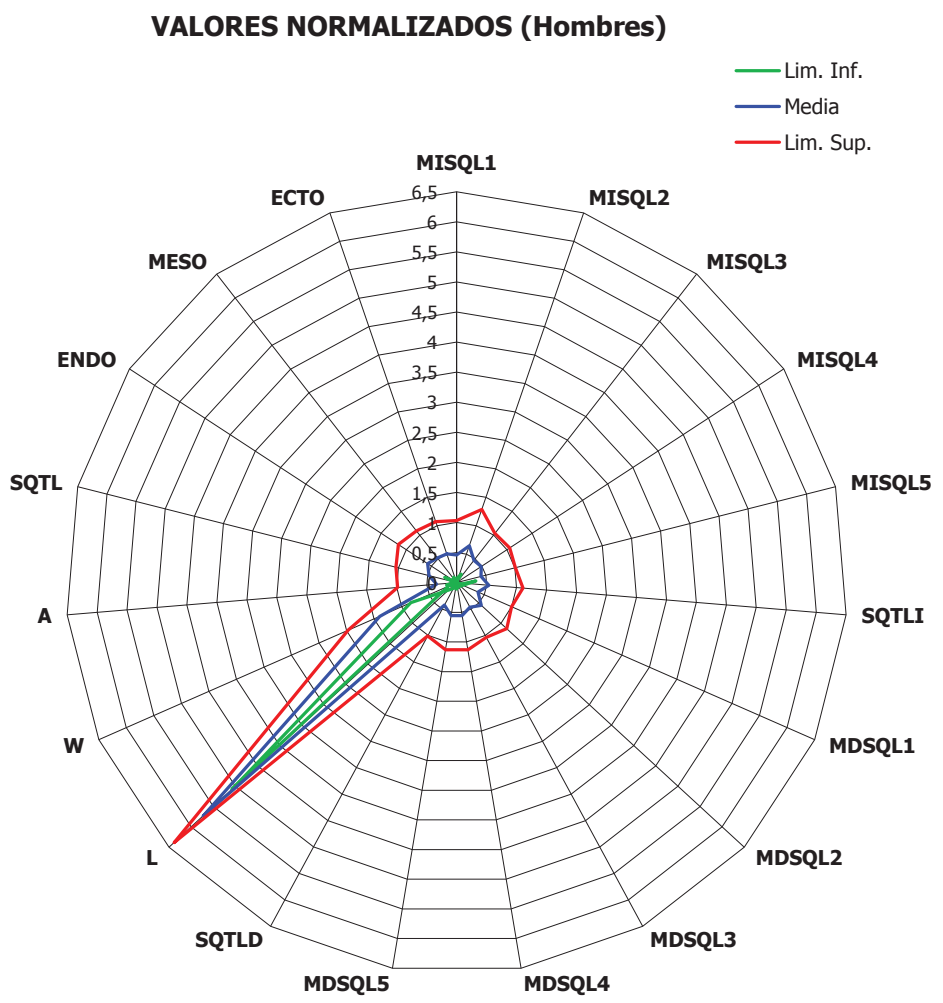

Gráfica 1. Radar con los valores normalizados: A. Damas; B. Hombres. MISQL= Mano Izquierda Sumatoria Líneas; MDSQL = Mano Derecha Sumatoria Líneas; SQTLI= Sumatoria Total Líneas Mano Izquierda; SQTLD= Sumatoria Total Líneas Mano Derecha; SQTL= Sumatoria Cantidad Total de Líneas; L= Presilla; W= Verticilo; A= Arco; Ecto, Meso \& Endo= Somatotipos. 


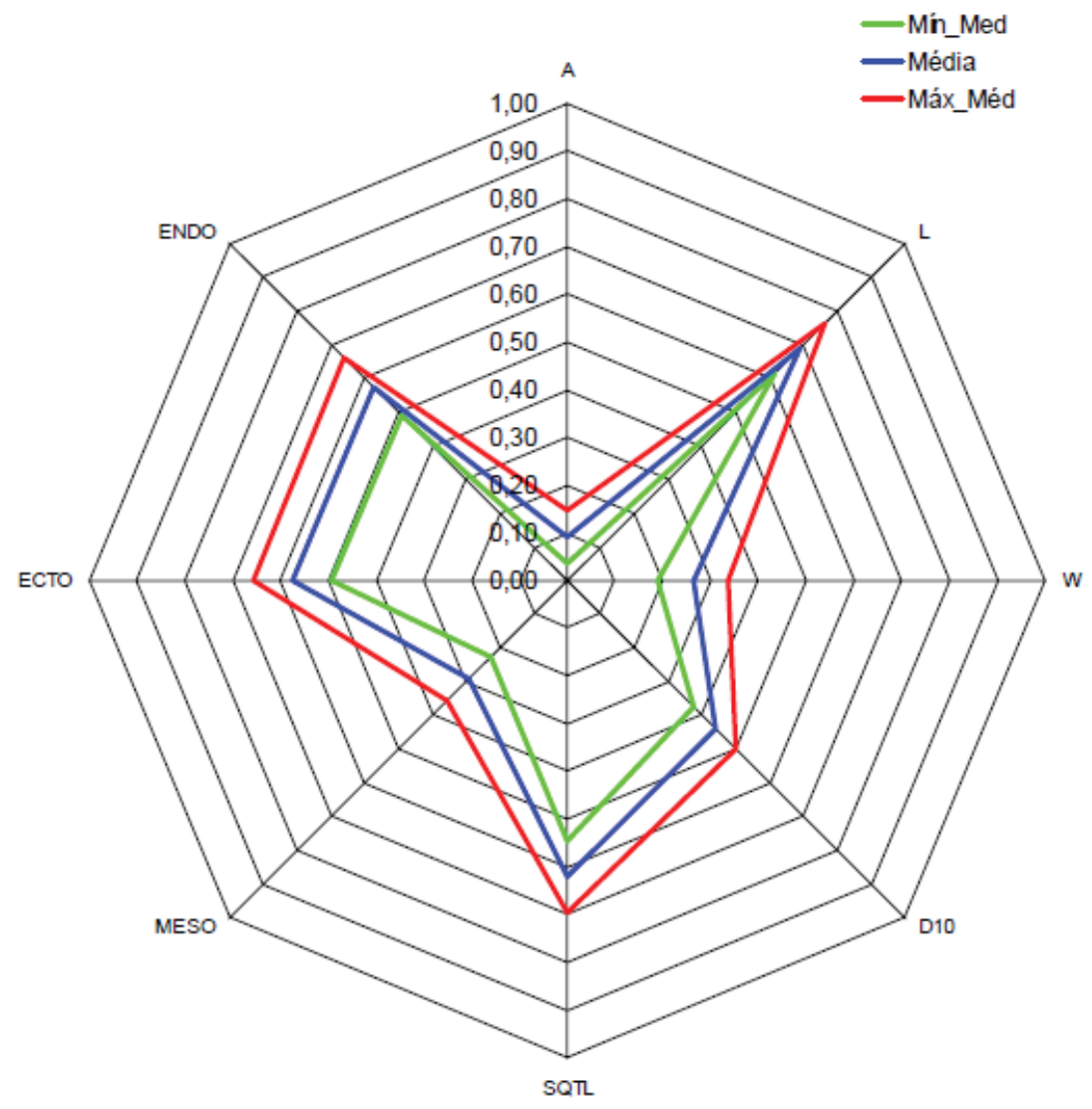

Gráfica 2: Fútbol de Campo Masculino de Alto Rendimiento, Río de Janeiro (Castanhede et al. 2003).

das las muestras presentadas hay ausencia de Arcos, lo cual, corrobora su nivel deportivo (Alto Logros), los deportes de conjunto (Fútbol y Voleibol), se caracterizan por una mayor presencia de Presilas y, en el deporte cíclico (Atletismo de Fondo), existe una equivalencia entre los diseños Presila y Verticilo. En lo referente a las variables cuantitativas, en el SCTL existe una similitud en las disciplinas discutidas, encontrándose en el nivel medio bajo y en el D10, en los deportes de pelota, se aprecian valores bajos, mientras en el atletismo su calificación es media alta. En cuanto al somatotipo, en deportes de conjunto, predomina un estado mesoectomorfo, presentándose diferencias, dependiendo de la posición que desempeñe el jugador y, en deportes cíclicos de larga duración, la composición corporal predominante será el ectomesomorfo, con un mínimo componente endomórfico. En cuanto a deportes de velocidad, se caracterizaron los patinadores venezolanos velocistas participantes en JJ.NN. (Lozano et al. 2006), de 2005, donde se encontró la predominancia en varones del somatotipo mesoendomorfo y, en damas, endomesomorfo.

Agradecimientos: Al Prof. Dr. José Fernandes Filho, por su apoyo en la normalización de los datos y la construcción de los radares; al Prof. Dr. Jaime Humberto Leiva Deantonio, por el apoyo bibliográfico; a la Universidad de Ciencias Aplicadas y Ambientales U.D.C.A, por el apoyo financiero para la realización de la siguiente investigación. Conflicto de Intereses: Los autores declaran que no existe ningún conflicto de intereses que ponga en riesgo la validez de los resultados presentados.

\section{BIBLIOGRAFÍA}

1. ABRAMOVA, T.; NIKITINA, T.; KOCHETKOVA, N. 2003. Orientación de la investigación científica en el laboratorio de antropología deportiva, morfología y genética del VNIIFK. Teoría Práct. Cult. Fís. 10:39-41.

2. ABRAMOVA, T.; NIKITINA, T.; OZOLIN, H. 1996. Posibilidades del empleo de la dermatoglífia dactilar en la selección deportiva. Teoría Práct. Cult. Fís. 3:8-14.

3. ABRAMOVA, T.; JDANOVA, A.; NIKITINA, T. 1990. Impresiones Dermatoglíficas y Somatotípo: Marcas de Constitución de Diferentes Niveles. Actualidades Mé- 
dicas y Antropológicas en el Deporte. Moscú. Cap. 2. p.94-95.

4. AGUDELO, C. 2012. Planificación del entrenamiento deportivo por modelación. Ed. Kinesis. Armenia. p.25-41.

5. BOUCHARD, C. 2007. Determinantes genéticos del rendimiento en resistencia. En: Shepard, R.; Astrand, P. La resistencia en el deporte. Ed. Paidotribo. Badalona, España. p.159-172.

6. CARTER, J. 2002. The Heath-Carter Anthropometric Somatotype - Instruction Manual. Department of Exercise and Nutritional Sciences. San Diego State University. p.2-3.

7. CARTER, J.; HEATH, B. 1990. Somatotyping - Development and Applications. Cambridge. Cambridge University Press. p.20-50.

8. CARVALHO, E.; FERNANDES FILHO, J.; NOVAES, J.S. 2005. Perfis dermatoglífico, somatotípico e fisiológico dos atletas de alto rendimento, participantes de corrida de resistência, no Rio de Janeiro. Fitness \& Perform. J. 4(3):168-174.

9. CASANOVAS, J. 2000. Enciclopedia visual de los deportes, Ed. Sport. Vol. 6, p.8-15.

10. CASTANHEDE, A.L.K.; DANTAS, P.M.S.; FERNANDES FILHO, J. 2003. Perfil dermatoglífico y somatotípico, de atletas de fútbol de campo masculino, de alto rendimiento em Rio de Janeiro - Brasil. Fitness \& Perform. J. 2(4):234-239.

11. CHAKRABORTY, R. 1991. The role of heredity and environment on dermatoglyphic traits. Dermatoglyphics: Science in transition. Birth Defects Original Article Series. 27(2):151-191.

12. CUMMINS, H.; MIDLO, C.H. 1963. Finger prints, palms and soles. An introduction to dermatoglyphics. Dover Publ. 319p.

13. DANTAS, E. 2012. La práctica de la preparación física. Ed. Paidotribo. Barcelona. p.227-383.

14. DE ANTON, F; DE LUIS, J. 2004. Sistema Inglés. En Policía científica. $4^{\mathrm{a}}$ ed. Valencia: tirant lo Blanh. p.423-459.

15. DOS SANTOS, L.; SILVA DANTAS, P.; FERNADES FILHO, J. 2007. Características genotípicas e fenotípi- cas em atletas velocistas. Rev. Desporto e Saúde da Fundação Técn. Cient. Desp. 4(1):49-56.

16. FERRÃO, M.L.D.; FILHO, J.F.; FORTES, M.S.R.; VIANA, M.V.; DANTAS, E.H.M. 2004. Efecto de la predominancia del tipo de fibra muscular sobre la pérdida de peso y el acondicionamiento aeróbico. Fitness \& Perform. J. 3(4):231-235.

17. FONSECA, C.L.T.; DANTAS, P.M.S.; FERNANDES, P.R.; FERNANDES FILHO, J. 2008. Perfil dermatoglífico, somatotípico e da força explosiva de atletas da seleção brasileira de voleibol feminino. Fitness \& Perform. J. 7(1):35-40.

18. GARCÍA MANSO, J. 2003. El talento deportivo: Formación de élites deportivas. Ed. Gymnos. p.17-28.

19. GROSSER, M.; BRÜGGEMANN, P.; ZINTL, F. 1990. Alto rendimiento deportivo: planificación y desarrollo. Ed. Martínez Roca. Barcelona. España. p.25-35.

20. GUZMÁN, C. 2003. Manual de criminalística, Buenos Aires: Ed. La Rocca. 624p.

21. HAHN, E. 1988. Entrenamiento con niños: teoría, práctica, problemas específicos. Ed. Martínez Roca. $165 p$.

22. JOÃO, A.; FERNANDES FILHO, J. 2002. Identificação do perfil genético, somatotípico e psicológico das atletas brasileiras de ginástica olímpica feminina de alta qualificação esportiva. Fitness \& Perform.J. 1(2):12-19.

23. LEIVA, J. 2010. Selección y orientación de talentos deportivos Ed. Kinesis. Armenia. p.71-76.

24. LOZANO, R.; CONTRERAS, D.; NAVARRO, L. 2006. Descripción antropométrica de los patinadores de velocidad sobre ruedas participantes en los Juegos Deportivos Nacionales de Venezuela, Diciembre de 2005. EFDeportes.com, Rev. Digital. Buenos Aires. 11(102). Disponible desde Internet en: http:// www.efdeportes.com/efd102/patin.htm (con acceso 08/03/2013).

25. MARLAND, S. 1972. La educación de los superdotados y talentosos: documentos Informe al Congreso de los Estados Unidos por el Comisionado de Educación de EE.UU. y de antecedentes presentados a la Oficina de Educación de EE.UU., 2 vols. Washington, DC: Imprenta del Gobierno de EE.UU. (Documentos de Gobierno Y4.L 02.11: G36). 
26. MARTÍNEZ LAGUNA, L.E.; TAMARIT MEDRANO, R.; RANGEL MAYOR, L. 2012. El empleo de marcadores genéticos en el proceso de selección de talentos. EFDeportes.com, Revista Digital. Buenos Aires. 17(171). Disponible desde Internet en: http:/www.efdeportes.com/efd171/marcadores-geneticos-en-seleccion-de-talentos.htm (con acceso 019/01/2013).

27. NISHIOKA, G.A.C.; DANTAS, P.M.S.; FERNANDES FILHO, J. 2007. Perfil dermatoglífico, somatotípico y de las cualidades físicas básicas de los bailarines becarios del Centro de Movimiento Deborah Colker. Fitness \& Perform. J. 6(5):331-337.

28. ROVER, C.; NODARI JUINIOR, R.J. 2012. Perfil dermatoglífico dos atletas participantes de modalidades coletivas dos Jogos Universitários Brasileiros - JUBs 2011. Unoesc \& Ciência - ACBS. 3(2):143-154.

29. RUIZ, L.; SÁNCHEZ, F. 1997. Rendimiento Deportivo. Claves para la Optimización de los Aprendizajes. Editorial Gymnos. Madrid. p.23-54.

30. SILVA. 2002. Diccionario básico del deporte y la educación física. Editorial Kinesis. Armenia, Colombia. p.41-42.

31. ZATSIORSKI, V. 1989. Metrología deportiva. Moscú, Editorial Planeta. p.278-287.

Recibido: Enero 14 de 2013

Aceptado: Abril 3 de 2013 